\title{
45-Gbps 3D-CAP Transmission over a 16-GHz Bandwidth SSMF Link assisted by Wiener Filtering
}

\author{
Lin Sun, Jiangbing Du*, Yue You, Chenyu Liang, Wenjia Zhang, Lin Ma and Zuyuan He
}

\begin{abstract}
In this study, the optical 3-dimensional carrier-less amplitude phase (3D-CAP) modulation up to 45 Gbps at $1550 \mathrm{~nm}$ is demonstrated and standard single mode fiber (SSMF) transmission up to $10-\mathrm{Km}$ is achieved. The 10-dB bandwidth of the optical back-to-back link is only $16 \mathrm{GHz}$. Digital Wiener filter is employed to compensate signal distortion with low algorithm complexity. The results indicate a power sensitivity of about 2-dBm after 10-Km transmission for achieving BER of 3.8e-3 at 7\% FEC limit. To our best acknowledgement, this is the highest transmission capacity for the 3D-CAP transmission over 10-Km SSMF at $1550 \mathrm{~nm}$. The demonstrated results of 3D-CAP modulation and transmission with paralleled data streams in 3 dimensions present a good potential for improving the density and reducing the cost of parallel optical transceivers in short reach optical interconnections.
\end{abstract}

Keywords—optical communication; modulation; carrier-less amplitude phase.

\section{Introduction}

In recent years, demands for data communication inside or between data centers and high performance computers are increasing rapidly, due to the non-stopping booming of information industry boosted by new technologies and services like Internet of Things (IoT), high definition video ( $2 \mathrm{~K}, 4 \mathrm{~K}$ and $8 \mathrm{~K}$ ), artificial intelligence (AI), Augmented Reality and Virtual Reality (AR/VR), 5G and so on. To meet the urgent demands of bandwidth, improved optical interconnection with large capacity, low power consumption and reduced complexity is highly desired. Several advanced modulation formats, like pulse amplitude modulation (PAM), carrier-less amplitude phase modulation (CAP) and discrete multi-tone (DMT) are recently exhibiting good potential for next generation optical interconnection to achieve $100 \mathrm{Gbps}$ or above per lane signal transmission with improved performance [1-6]. Among them, PAM-4 is widely studied and it has shown good performance with rather simple configuration and low system complexity, although the performance of spectrum efficiency (SE) and robustness to optical dispersion are quite limited, compared with CAP and DMT. And on the other hand, Nyquist-PAM4 based on pulse shaping can slightly improve its dispersion tolerance [7-8]. DMT, based on OFDM theory with the use of adaptively bit allocation according to channel response, provides improved tolerance to fiber dispersion and enlarged transmission capacity [9-10]. However, due to the complex storage and calculation like IFFT, serial-to-parallel transform 
(SPT) and bit loading algorithm, the power consumption and latency of DMT are very large and thus limit the practical application of it.

1

Meanwhile, CAP offers a balanced performance of high SE combined with moderate complexity [9-15]. It performs orthogonal multiplexing based on signal pulse shaping and matched filtering without additional carrier in need, realizing cost-effective intensity modulation and direct detection (IM-DD). And the matched filters, as the key of CAP modulation, can be implemented by analogue circuits, shown great application potential [16].

Previous works of CAP mainly concentrate on the conventional two-dimensional CAP and Multiband-CAP. Among them, a high SE of $4 \mathrm{bit} / \mathrm{s} / \mathrm{Hz}$ is reported in WDM-CAP-PON [14] but the data rate is only $1.25 \mathrm{Gbps}$. And 40-Gbps conventional CAP-32 signals are transmitted over 80-km SMF employing least mean square (LMS) algorithm [15]. On the other hand, Multiband-CAP, with the capability to load signals with different sensitivity to different frequency bands, can achieve improved transmission performance. Single-lambda 100-Gbps-plus generation of multiband-CAP has been demonstrated both in SMF system and MMF system [9, 17].

In addition, CAP modulation can easily scale to multiple dimensions based on finite-impulse-response (FIR) filter design, thus provides enhanced communication flexibility, such as three-dimensional CAP (3D-CAP) and four-dimensional CAP (4D-CAP) $[10,18]$. In a 3D-CAP scheme, three independent data streams are multiplexed and de-multiplexed through the digital matched filters. The data streams of 3D-CAP keep paralleled all the time without SPT, FFT, IFFT, storage, or any other signal processing processes. Therefore, multi-dimensional CAP is particularly suitable for parallel optical interconnections (e.g. four-lane quad-small-form-pluggable QSFP) by orthogonal multiplexing of the paralleled data streams to reduce the cost and increase the capacity with low complexity and latency. To date, the related works are mostly about its application in different applied fields [19,20] and performance evaluation [21].

In this study, we demonstrated optical 3D-CAP signal generation up to $45 \mathrm{Gbit} / \mathrm{s}$ and SSMF transmission over 10-km. The optical back-to-back link in the experiment has a 10-dB bandwidth of only 16-GHz. At the receiver side, Wiener filter, a much simpler DSP process than LMS, is employed for the compensation. With the use of Wiener filter, bit error ratio (BER) below 7\%FEC is achieved with received optical power of 2-dBm. To our best acknowledgement, this is the highest experimental transmission capacity of 3D-CAP signal.

\section{Experimental Setup}

The 3D-CAP optical transmission setup is illustrated in Fig. 1. The digital 3D-CAP16 signal is generated by the sum of three up-sampled PAM-4 signals with FIR pulse shaping on the Matlab platform. To design the matched filters for CAP modem system, the optimization problem at frequency domain is solved using Minimax algorithm [22]. The minimax algorithm gives limitation to frequency responses of three shaping filters. The optimization expression is stated in Eq. 1 to select three sequences $\left\{f_{0}, f_{1}, f_{2}\right\}$ with the given frequency magnitude response, and the linear constraint $F_{T_{x}} F_{R x}=z^{-n} I$ aims to obtain the corresponding receiver matched filters. In Eq. 1, H is the frequency magnitude responses of filters, $\mathrm{R}$ is the required bandpass response, $\mathrm{F}_{\mathrm{Tx}}$ and $\mathrm{F}_{\mathrm{Rx}}$ are $\mathrm{z}$ transformations of the transmitter filter and receiver filter respectively, $\mathrm{z}^{-\mathrm{n}}$ means $\mathrm{n}$ delay elements of the transmission channel. Under the linear constraint, it can always achieve the perfect reconstruction (PR) condition to separate the data streams without crosstalk. 


$$
\min _{\left\{f_{0}, f_{1}, f_{2}\right\}}\{\max (|H-R|)\} \text { subject to } F_{T x} F_{R x}=z^{-n} I
$$

1 The frequency responses of selected filters are shown in Fig.2. In order to guarantee enough high transmission capacity, the 3 filter length in this experiment is set as low as 4, equals to the up-sampling factor. The correlation curves of matched filters 4

Fig. 1 Experimental setup. AWG: Arbitrary waveform generator, SSMF: Standard single-mode fiber, PD: Photodiode, DSO: Digital storage oscilloscope. MZM: Mach-Zehnder modulator, TIA: Trans-impedance amplifier, PC: Polarization controller.

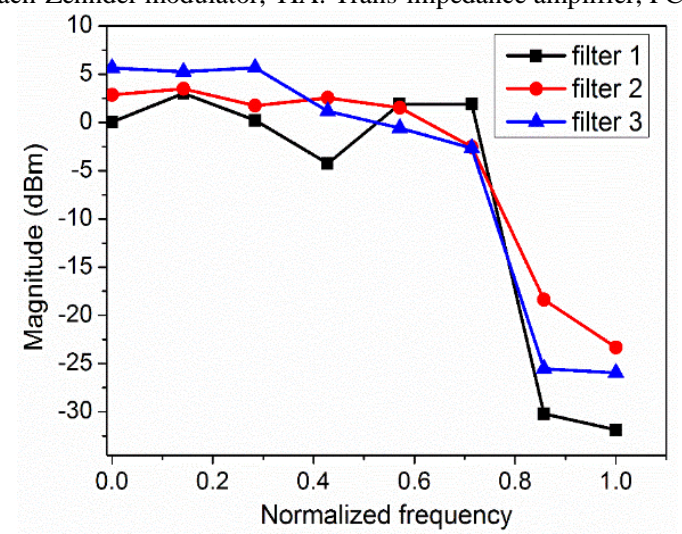

Fig. 2. Frequency responses of shaping filters with 15 taps. 

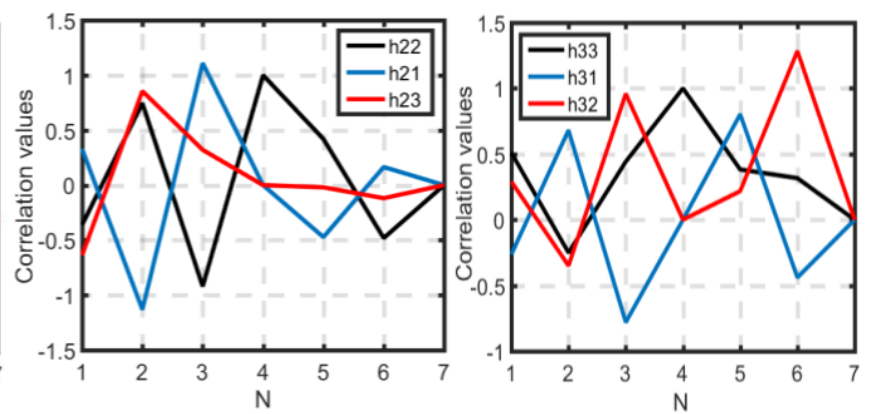

Fig. 3. Correlation curves of matched filters with 4 taps.

An 8-bit arbitrary waveform generator (AWG) with bandwidth of $25-\mathrm{GHz}$ is employed to convert the digital 3D-CAP signal to analog mode. Because AWG is working at the fixed sampling rate of $64 \mathrm{Gsa} / \mathrm{s}$, the up-sampling and holding process with factor of 2.13 is required to obtain 30-Gbaud/s electrical signal. Due to the fact that up-sampling factor is 4, the bit rate of PAM-4 at every dimension is $15 \mathrm{Gbps}$, so total data rate of 3D-CAP signal is $45 \mathrm{Gbps}$. Due to the limitation of Vpp of AWG's output at 500 $\mathrm{mV}$, a linear amplifier (SHF806E) is used to boost the signal power, effectively driving optical modulator. In addition, the pre-distortion process is embedded in AWG to mitigate the fading effect of fiber channel. A 17-GHz commercial MZ-M is used to modulate the continuous light at $1550.5 \mathrm{~nm}$, provided by NKT tunable laser, and the typical extinction ratio of the modulator is over $20 \mathrm{~dB}$. The optical spectrum of modulated light is shown in Fig. 4 (a). Then the modulated lightwave is transmitted over a spool of SSMF. At the receiver side, a $25-\mathrm{GHz}$ photodiode with $0.7-\mathrm{A} / \mathrm{W}$ responsibly is used to detect optical signal. The electrical spectrum of detected 3D-CAP signal in optical B2B case is measured by electrical spectrum analyzer (ESA), shown in Fig. 4 (b), the efficient bandwidth is $15 \mathrm{GHz}$, indicating that the 3D-CAP16 signal has the SE value of $3 \mathrm{bit} / \mathrm{s} / \mathrm{Hz}$. A 59-GHz digital storage oscilloscope (DSO) (Keysight Z592A) with 160-Gsa/s sampling rate is used to sample the signal to digital mode, followed by offline DSP. In addition, the frequency responses of fiber channels are measured by multi-tone signal generated by AWG, shown in Fig. 5. The results show that optical back-to-back link has only $16-\mathrm{GHz} 10-\mathrm{dB}$ bandwidth during this experiment.
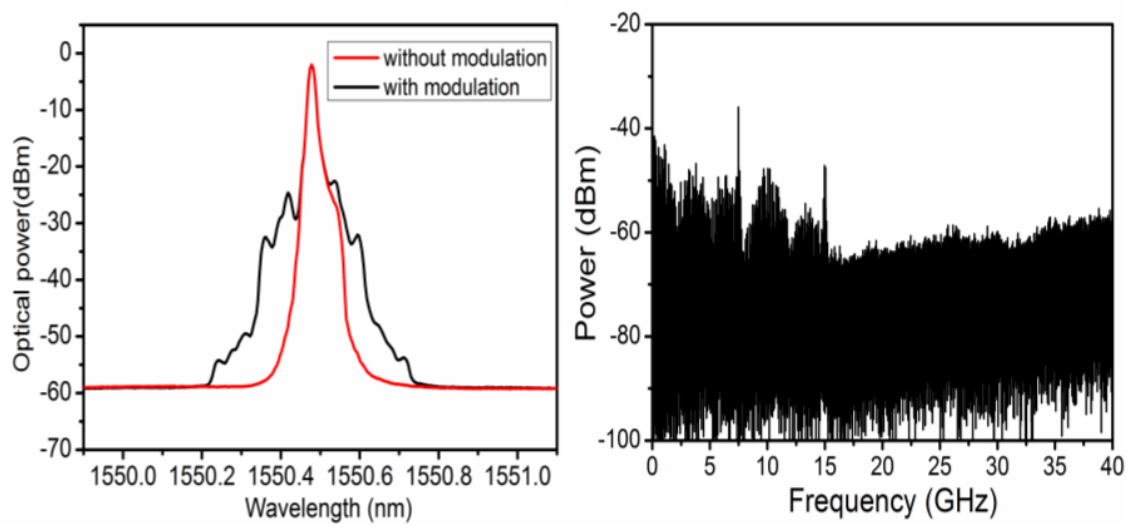

Fig. 4 (a) optical spectrums, (b) Electrical spectrum of detected 3D-CAP signal after PD.

The offline DSP includes linear interpolation, resampling, matched filtering. Interpolation process is always required in such offline BER measurement systems, to reduce the measurement deviation in digital domain [23]. In this experiment, linear interpolation with factor of 10 is used to increase the sampling rate, ensuring the reliability of signal recovery and BER measurement. And resampling process before matched filtering is performed to demodulate 3D-CAP signal. The 3D-constellation 
of 3D-CAP signal with received optical power of 2-dBm in the case of B2B is shown in Fig. 6, as well as the planar constellations.

After 10-km SMF transmission, the constellations of detected signal are shown in Fig. 7. The constellations of 3D-CAP signals

1

2

3

4

5

6

7

8

9

10

11

12

13

14

15

16

17

18

19

20

21

22

23

24

25

26

27

28

29

30

31

32

33

34

35

36

37

38

39

40

41

42

43

44

45

46

47

48

49

50

51

52

53

54

55

56

57

58

59

60

61

62

63

64

65

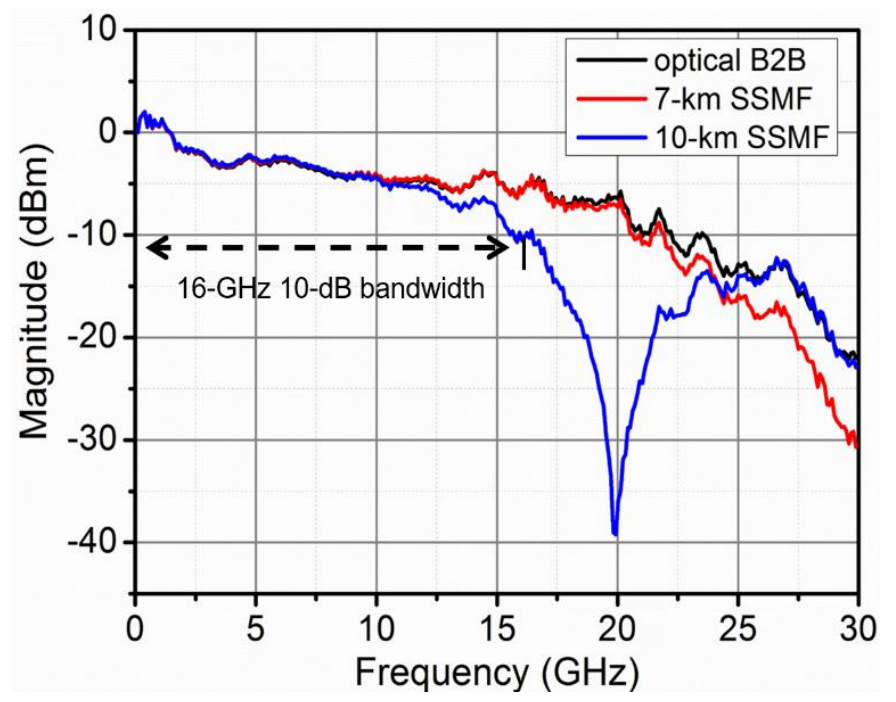

Fig. 5. Frequency responses of fiber channel.
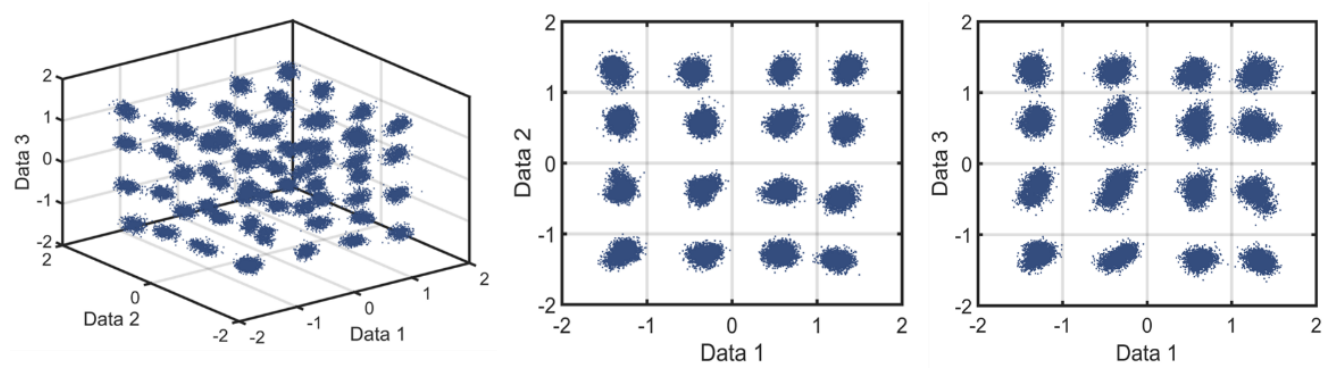

Fig. 6. 3D-CAP constellations in the case of B2B.
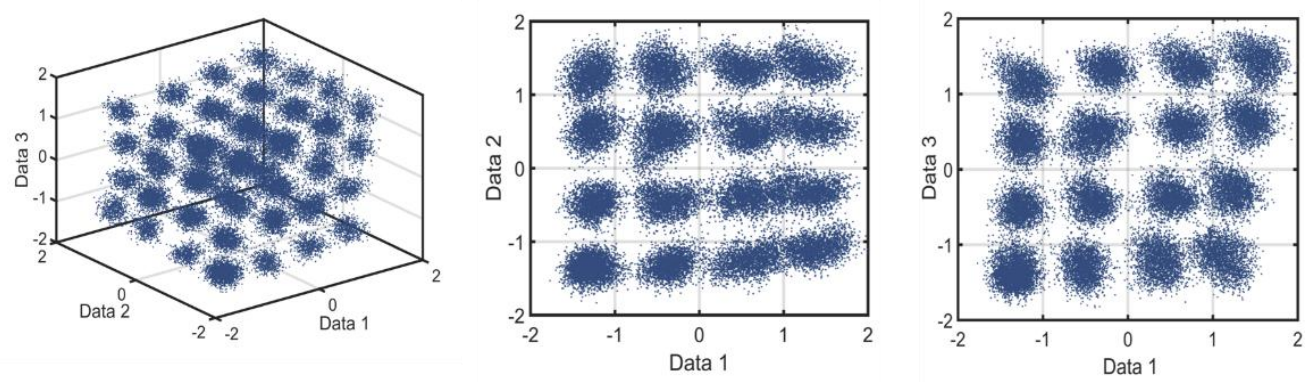

Fig. 7. 3D-CAP constellations after 10-km SMF transmission.

In order to improve the signal quality, Wiener filter is employed to compensate the signal distortion in the receiving side. Wiener filter is an optimum filter based on mean square error algorithm [24]. The schematic diagram of Wiener filter is shown in Fig. 8. To obtain the optimal tap of Wiener filter, the known training sequence $d(n)$ is to be sent into system, and the corresponding sampled output is $r(n)$. The tap of Wiener filter is directly calculated by the matrix product of cross-correlation matrix of $d(n)$ and $r(n)$, and the invers of self-correlation matrix of $d(n)$. Different from LMS filter, it does not require optimal selection of 
convergence step, as the results, it is desired to be advantageous over LMS with low computing complexity and stable filter performance.

1

2

3

4

5

6

7

8

9

10

11

12

13

14

15

16

17

18

19

20

21

22

23

24

25

26

27

28

29

30

31

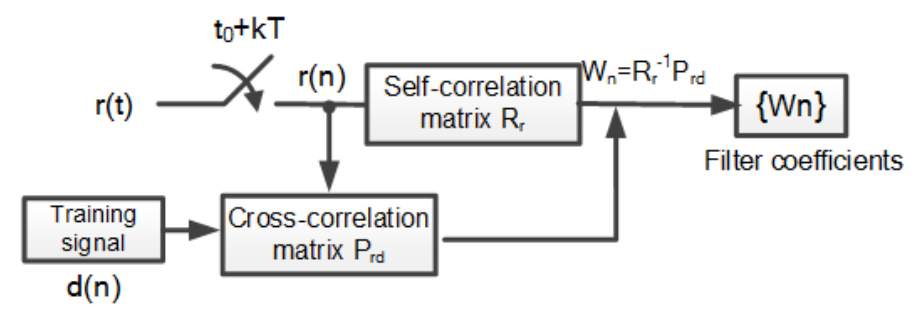

Fig. 8. Schematic diagram of Wiener filter in receiving side.

So we choose Wiener filter to do the signal equalization in the receiving side. Firstly, we use the sampled 3D-CAP data with $3-\mathrm{dBm}$ received power to calculate the coefficients of Wiener filter. The length of training sequence is 1016 and the length of Wiener filter set as 635. Because the tap coefficients of Wiener filter are directly calculated by production of the correlation matrixes, the filter length is linearly relevant to the length of statistical sequences. In order to obtain enough accuracy in statistic process, similar to FFT function, the sequence length has to be long enough. However, one can reduce the tap number of Wiener filter through down-sampling in frequency domain.

We measure the BER values in the case of optical back-to-back, after 7-km transmission and after 10-km transmission, with changeable received optical power. The BER curves of 3D-CAPs are shown in Fig. 9. The results indicate that the Wiener filter can reduce near one order value of BER. And the BER below 3.6e-3 is achieved using Wiener filter with 2-dBm received optical power.

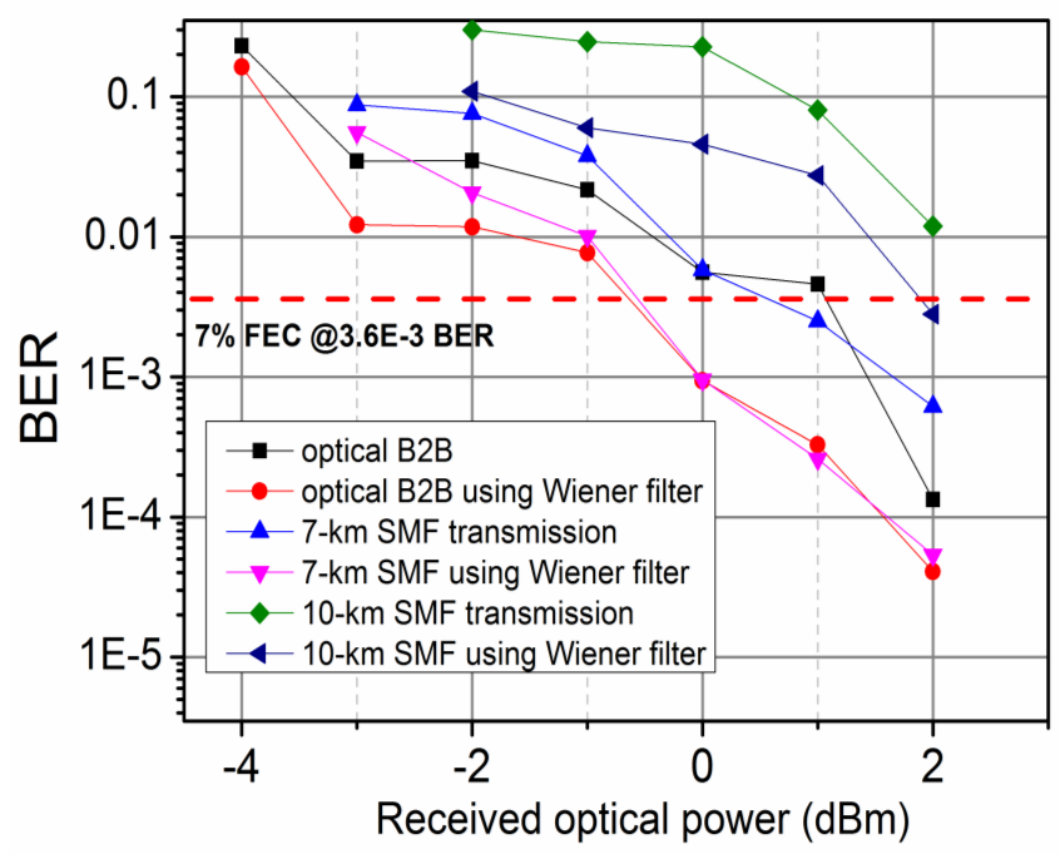

Fig. 9. BER curves of 3D-CAP signals.

\section{Conclusion}


In this work, we successfully transmit 45-Gbit/s optical 3D-CAP signal over 10-km SMF using Wiener filter as the equalizer. To our best acknowledgement, this is the highest transmission capacity of optical 3D-CAP signal. And the paralleling

1

2

communication with low cost and power consumption based on multi-dimensional CAP can surely be applied in paralleling optical interconnection, with only one pair of laser and photodiode in use.

\section{ACKNOWLEDGMENT}

The work was supported by Natural National Science Foundation of China (NSFC) (61675128, 61307107); Shanghai STCSM Science Foundation (15511103102, 13ZR1456200).

\section{REFERENCES}

[1] Chao Yang, Rong Hu, Ming Luo, Qi Yang, Cai Li, Haibo Li, Shaohua Yu "Dense wavelength division multiplexing networks: principle and applications,” IEEE Photon. J. 8(3) (2016).

[2] Rafael Rios-Müller, Jeremie Renaudier, miquel mestre, Haik Mardoyan, Agnieszka Konczykowska, Filipe Jorge, Bernadette Duval, and Jean-Yves Dupuy "Multi-Dimension Coded PAM4 Signaling for 100Gb/s Short-Reach Transceivers," Optical Fiber Communication Conference, Th1G.4 (2016).

[3] Miguel Iglesias Olmedo, Tianjian Zuo, Jesper Bevensee Jensen, Qiwen Zhong, Xiaogeng Xu, Sergei Popov, Idelfonso Tafur Monroy "Multiband Carrierless Amplitude Phase Modulation for High Capacity Optical Data Links,” J. Lightwave. Technol. 32(4), 798-804 (2014).

[4] Jinlong Wei, Qixiang Cheng, David G. Cunningham, Richard V. Penty, and Ian H. White "100-Gb/s Hybrid Multiband CAP/QAM Signal Transmission Over a Single Wavelength,” J. Lightwave. Technol. 33(2), 415-423 (2015).

[5] Toshiki Tanaka, Masato Nishihara, Tomoo Takahara, Weizhen Yan, Lei Li, Zhenning Tao, Manabu Matsuda, Kazumasa Takabayashi, and Jens Rasmussen "Experimental Demonstration of 448-Gbps+ DMT Transmission over 30-km SMF," Optical Fiber Communication Conference, M21.5 (2014).

[6] Kangping Zhong, Xian Zhou, Tao Gui, Li Tao, Yuliang Gao, Wei Chen, Jiangwei Man, Li Zeng, Alan Pak Tao Lau, and Chao Lu "Experimental study of PAM-4, CAP-16, and DMT for $100 \mathrm{~Gb} / \mathrm{s}$ Short Reach Optical Transmission Systems," Opt. Express. 23(2), 1176-1189 (2015).

[7] Nobuhiko Kikuchi, Riu Hirai "Intensity-modulated / direct-detection (IM/DD) Nyquist pulse-amplitude modulation (PAM) signaling for 100-Gbit/s/ $\lambda$ optical short-reach transmission," The European Conference on Optical Communication (ECOC) (2014).

[8] Hiroshi Yamazaki, Munehiko Nagatani, Shiaeru Kanazawa, Hideyuki Nosaka, Toshikazu Hashimoto, Akihide Sano, Yutaka Miyamoto "160-Gbps Nyquist PAM4 transmitter using a digital-preprocessed analog-multiplexed DAC," The European Conference on Optical Communication (ECOC) (2015).

[9] Lin Sun, Jiangbing Du, and Zuyuan He, "Multiband 3-Dimensional Carrierless Amplitude Phase Modulation for Short Reach Optical Communications,” J. Lightwave. Technol. 34(13), 3103-3109 (2016).

[10] Lin Sun, Jiangbing Du, and Zuyuan He, "Experimental demonstration of 30-Gbit/s 3D-CAP modulation for short reach optical interconnection," Optical interconnects Conference (2016). 
[11] Jonathan D. Ingham, Richard Penty, Ian White, and David Cunningham, "40 Gb/s Carrierless Amplitude and Phase Modulation for Low-Cost Optical Datacommunication Links," Optical Fiber Communication Conference OThz3 (2011).

1

[12] Junwen Zhang, Jianjun Yu, Fan Li, Nan Chi, Ze Dong, and Xinying Li, "11 $\times 5 \times 9.3 \mathrm{~Gb} / \mathrm{s}$ WDM-CAP-PON based on optical single-side band multi-level multi-band carrier-less amplitude and phase modulation with direct detection" Opt. Express. 21(16), 18842-18848 (2013).

[13] Li Tao, Yiguang Wang, Yuliang Gao, Alan Pak Tao Lau, Nan Chi, and Chao Lu, "Experimental demonstration of 10 $\mathrm{Gb} / \mathrm{s}$ multi-level carrier-less amplitude and phase modulation for short range optical communication systems" Opt. Express. 21(5), 6459-6465 (2013).

[14] Roberto Rodes, Marcin Wieckowski, Thang Tien Pham, Jesper Bevensee Jensen, Jarek Turkiewicz, Jerzy Siuzdak, and Idelfonso Tafur Monroy, "Carrierless amplitude phase modulation of VCSEL with $4 \mathrm{bit} / \mathrm{s} / \mathrm{Hz}$ spectral efficiency for use in WDM-PON" Opt. Express. 19(27), 26551-26556 (2011).

[15] Yuliang Gao, Qunbi Zhuge, Wei Wang, Xian Xu, Jonathan M. Buset, Meng Qiu, Mohamed Morsy-Osman, Mathieu Chagnon, Feng Li, Liang Wang, Chao Lu, Alan Pak Tao Lau, and David V. Plant, "40 Gb/s CAP32 short reach transmission over 80 km single mode fiber” Opt. Express. 23(9), 11412-11423 (2015).

[16] J. L. Wei, D. G. Cunningham, R. V. Penty, and I. H. White, "Study of 100 Gigabit Ethernet Using Carrierless Amplitude/Phase Modulation and Optical OFDM,”J. Lightwave. Technol. 31(9), 1367-1373 (2013).

[17] Rafael Puerta, Mikel Agustin, Lukasz Chorchos, J. Tonski, J.-R Kropp, N. Ledentsov, V. A. Shchukin, N. N. Ledentsov, R. Henker, Idelfonso Tafur Monroy, Juan José Vegas Olmos, and J.P. Turkiewicz “107.5 Gb/s 850 nm multiand single-mode VCSEL transmission over 10 and 100 m of multi-mode fiber," Optical Fiber Communication Conference, Th5B.5 (2016).

[18] Maisara Binti Othman, Xu Zhang, Lei Deng, Marcin Wieckowski, Jesper Bevensee Jensen, Idelfonso Tafur Monroy, "Experimental Investigations of 3-D-/4-D-CAP Modulation With Directly Modulated VCSELs," IEEE Photon. Technol. Lett. 24(22), 2009-2012 (2012).

[19] Xu Zhang, Maisara B. Othman, Xiaodan Pang, Jesper Bevensee Jensen, and Idelfonso Tafur Monroy "Bi-directional Multi Dimension CAP Transmission for Smart Grid Communication Services," Asia Communications and Photonics, AS3C.4 (2012).

[20] Grzegorz Stepniak, "Three-Dimensional CAP for Short-Reach Bandwidth-Limited Optical Connections," J. Lightwave. Technol. 32(14), 2516-2523 (2014).

[21] Grzegorz Stepniak, "Comparison of Efficiency of N-Dimensional CAP Modulations," Signal Processing in Photonic Communications, ST1D.3 (2014).

[22] A. F. Shalash, K. K. Parhi , "Multidimensional carrierless AM/PM systems for digital subscriber loops," Trans. on. Commun. 47(11), 1655-1667 (1999).

[23] Steffen Freund, Hadrien Louchet, Gerard Schuetzler, Michael Rohde, André Richter "Investigation of DSP Requirements for Coherent Transmission using Experimental 10Gbaud/s 8PSK Data," The European Conference on Optical Communication (ECOC) (2015).

[24] J. S. Goldstein, I. S. Reed, L. L. Scharf, "A multistage representation of the Wiener filter based on orthogonal projections," Trans. on. Inform. 47(11), 1655-1667 (1999). 